\title{
Which method is more suitable for advanced gastric cancer with enlarged lymph nodes, laparoscopic radical gastrectomy or open gastrectomy?
}

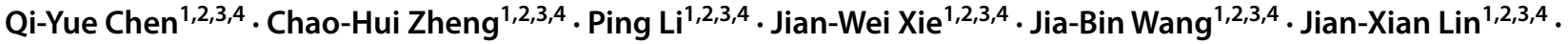 \\ Jun Lu ${ }^{1,2,3,4}$. Long-Long Cao ${ }^{1,2,3,4} \cdot$ Mi Lin $^{1,2,3,4} \cdot$ Ru-Hong Tu ${ }^{1,2,3,4} \cdot$ Ze-Ning Huang ${ }^{1,2,3,4} \cdot$ Ju-Li Lin Li,2,3, $_{\text {. }}$ \\ Chang-Ming Huang 1,2,3,4
}

Received: 30 March 2017 / Accepted: 22 January 2018 / Published online: 30 January 2018

(c) The International Gastric Cancer Association and The Japanese Gastric Cancer Association 2018

\begin{abstract}
Background The oncologic outcome of laparoscopic radical gastrectomy (LG) with open radical gastrectomy (OG) for gastric cancer with preoperative enlarged lymph nodes (LNs) remains ambiguous.

Methods The long-term outcomes of LG $(n=855)$ versus OG $(n=154)$ in gastric adenocarcinoma patients were analyzed retrospectively. Patients were stratified according to enlarged $(>10 \mathrm{~mm})$ and small $(\leq 10 \mathrm{~mm}) \mathrm{LNs}$ (ELN and SLN) based on the long-axis diameter of the LNs.

Results The violin plot indicates that the distribution of ELN size was similar between two groups. Survival curves demonstrated that the overall survival (OS) in LG is enhanced compared with OG $(p=0.034)$. A stratified analysis revealed that the OS was better in the LG group compared with the OG group for patients with ELNs $(p=0.038)$. In a forest map analysis, the actual 3-year OS rate for LG was significantly increased compared with OG in enlarged suprapancreatic LN (ESLNs) patients. Stratified analysis based on different diameters of ESLNs revealed that the actual 3-year OS and cancerspecific survival (CSS)rate for LG was significant increased compared to OG with a diameter from 1.0 to $1.9 \mathrm{~cm}$ for pII or pIII stage patients. However, the actual 3-year OS and CSS rate for LG was significantly reduced compared with OG when the size exceeded $2.5 \mathrm{~cm}$.

Conclusions For advanced gastric cancer with an ESLN diameter from 1.0 to $1.9 \mathrm{~cm}$, LG could be chosen preferentially; nevertheless, LG is not safe if ESLN are $>2.5 \mathrm{~cm}$.
\end{abstract}

Keywords Gastric carcinoma $\cdot$ Enlarged LN $\cdot$ Lymphadenectomy $\cdot$ Long-term outcome $\cdot$ Suprapancreatic area

Electronic supplementary material The online version of this article (https://doi.org/10.1007/s10120-018-0800-7) contains supplementary material, which is available to authorized users.

Chang-Ming Huang

hcmlr2002@163.com

1 Department of Gastric Surgery, Fujian Medical University Union Hospital, No. 29 Xinquan Road, Fuzhou 350001, Fujian Province, China

2 Department of General Surgery, Fujian Medical University Union Hospital, Fuzhou, China

3 Key Laboratory of Ministry of Education of Gastrointestinal Cancer, Fujian Medical University, Fuzhou, China

4 Fujian Key Laboratory of Tumor Microbiology, Fujian Medical University, Fuzhou, China

\section{Introduction}

Lymph node metastasis (LNM) is the most common pattern of metastatic spread in gastric cancer [1-3]. Therefore, gastric cancer, especially in advanced stages, presents with enlarged lymph nodes (ELNs). An ideal curative operation for gastric cancer can only be achieved via the complete dissection of perigastric LNs, including ELNs. Since the first reported laparoscopic gastrectomy for early gastric cancer by Kitano in 1994 [4], this technical approach has developed rapidly because its minimally invasive nature confers advantages over open radical gastrectomy (OG), including reduced blood loss, reduced pain, an accelerated recovery, and better cosmetic results; laparoscopic gastrectomy provides not only favorable short-term outcomes but also equivalent oncologic outcomes [5-10]. 
Improvements in laparoscopic techniques and surgical instruments have led to the development of laparoscopicassisted gastrectomy with lymphadenectomy for gastric cancer. Accordingly, laparoscopic radical gastrectomy (LG) has been increasingly performed for advanced gastric cancers with good LN dissection efficacy and short-term outcomes as reported by several retrospective studies [11-13]. However, given the lack of large-scale data concerning the longterm outcomes for these patients, the use of laparoscopic gastrectomy for the treatment of advanced gastric cancer remains a contentious issue.

ELNs increase the difficulty of lymphadenectomies for gastric cancer, and whether LG can achieve optimal longterm oncologic results for gastric cancer with ELNs has not been determined to date. Moreover, to the best of our knowledge, no study has compared the long-term results of LG and OG in patients with ELNs. This delay hampers the advancement and expanded use of the laparoscopic approach in the treatment of more advanced stages of gastric cancer. Consequently, before laparoscopy can become a universally applicable surgery for most patients with ELNs, the analysis of these long-term outcomes is required. Therefore, the aim of this study was to evaluate the long-term oncologic outcomes of LG for gastric cancer patients with preoperative ELNs by comparing them with those of patients who underwent $\mathrm{OG}$ by analyzing the long-term follow-up data of a large number of patients from our center. This information provides a reference for clinical practice before prospective studies confirm these data.

\section{Materials and methods}

\section{Patients}

From December 2009 to May 2012, a retrospectively maintained database identified 1081 patients with gastric cancer who underwent radical gastrectomy with the same surgical team with more than $1000 \mathrm{OG}$ and $500 \mathrm{LG}$ experiences at Fujian Medical University Union Hospital in China. Patient demographics, underlying diseases, data on surgery, and data on preoperative and postoperative monitoring including complications and the regular follow-up results were recorded into this clinical database system by the research assistant periodically, which was reviewed and verified by the senior doctors. Radical gastrectomy requires an adequate margin of normal tissue around the tumor, resection of at least two-thirds of the stomach, dissection of perigastric lymph nodes, and en-bloc removal of organs involved by direct extension. In this study, 35 non-radical gastrectomies were excluded in the same time. Patients with T4b $(n=57)$, remnant gastric cancer $(n=14)$ and splenectomy due to ELNs at the splenic hilus $(n=1)$ were also excluded. In total,
1009 patients were included in this study. Of these patients, 855 patients underwent LG, and 154 patients underwent OG. The rate of R1 resection due to pathological margin in LG and OG groups was similar [1.4\% (13/917) vs. $2.2 \%$ (4/181), $P=0.438]$. The diameter of each LN was measured using transverse multidetector-row computed tomography (MDCT) images and was recorded by two experienced radiologists. The slice thickness of MDCT was $0.625-2.5 \mathrm{~mm}$ according to different equipment. LNs with a long-axis diameter greater than $10 \mathrm{~mm}$ were regarded as clinically metastasized according to the Refs. [14-16]. Patients were stratified by large $(>10 \mathrm{~mm})$ and small $(\leq 10 \mathrm{~mm}) \mathrm{LNs}$ (ELN and SLN) based on the long-axis diameter of the LNs as measured preoperatively. Patients who diagnosed with cT1 to cT4a and without clinical evidence of distant metastases were informed of the possible complications of the procedure and the advantages and disadvantages of a laparoscopic compared with an open approach. Written informed consent was obtained from all patients prior to the operation. As for the cases with an enlarge LNs, they were all presented at multi-disciplinary rounds in our institution. This study was approved by the institutional review board of Fujian Medical University Union Hospital (Approval number: 20070428).

Preoperative imaging studies were routinely performed following endoscopic examination and upper gastrointestinal contrast to confirm the tumor location, including chest radiography, computed tomography (CT) scanning, and ultrasonography (US) of the abdomen to evaluate the diameter of LNs. Bone scanning and PET-CT were not a routine examination for all the patients, but only performed for the patients with clinical suspicion of distant metastasis in our center. Staging was determined according to the 7 th edition of the International Union Against Cancer (UICC)'s TNM classifications [17]. Postoperative follow-ups were performed every 3 months for 2 years and then every 6 months from years 3 to 5. Adjuvant chemotherapy with 5-fluorouracil (5-FU)-based regimens (mostly 5 -FU with cisplatin) was recommended to most patients with advanced gastric cancer.

The inclusion criteria were as follows: histologically confirmed gastric cancer based on the analyses of endoscopic biopsy specimens; pathologically confirmed stage $\mathrm{T} 1-\mathrm{T} 4 \mathrm{a}$; no evidence of distant metastasis by means of the aforementioned examinations; no evidence of adjacent organ invasion (pancreas, spleen, liver, colon, etc.) or obvious ELNs around the abdominal aorta; and completion of curative R0 according to the pathological diagnosis after surgery. The exclusion criteria included the following: remnant gastric cancer; intraoperative evidence of peritoneally disseminated or distant metastasis; pathologically confirmed T4b stage or combined major organ resection; incomplete pathological data; combined splenectomy due to enlarged splenic hilar LNs; and conversion to open surgery. A conversion 
to open laparotomy was required in 5 patients $(0.58 \%)$. The reasons for conversion were uncontrolled diffuse bleeding in the operation field for 3 cases, abdominal adhesions for 1 patient, and peripheral viscera injuries in 1 patient.

\section{Variable definitions}

It is quite difficult to accurately confirm which nodal group the ELNs belong to prior to operation. Thus in this study, the ELNs were divided into five areas according to their approximate anatomical location. Specifically, No. $14 \mathrm{v}$ and No. 6 LNs were considered to be in area I. No. 7, No. 8a, No. 9, No. 11p, No. 12a and No. 5 LNs were considered to be in area II. No. 1 and No. 3 LNs were considered to be in area III. No. 4 LNs were considered to be in area IV. Recurrences were classified as locoregional (nodal or gastric), peritoneal, hematogenous (e.g., liver, lung, bone, etc.) or combined.

\section{Surgical procedures}

The OG procedure was performed in a standard fashion, through a midline incision. The sequence of the lymphadenectomy of LAG was as follows. (1) For distal gastrectomy: No. $6 \rightarrow$ Nos. 7, 9, 11p $\rightarrow$ No. 3, $1 \rightarrow$ Nos. 8a, 12a, $5 \rightarrow$ No. 4sb. For (2) total gastrectomy: No. $6 \rightarrow$ Nos. 7 , $9,11 \mathrm{p} \rightarrow$ Nos. 8 a, $12 \mathrm{a}, 5 \rightarrow$ No. $1 \rightarrow$ No. $4 \mathrm{sb} \rightarrow$ Nos. 10 , $11 \mathrm{~d} \rightarrow$ No. 2. For details, please see the references [18-21].

\section{Follow-up}

Postoperative follow-ups were performed every 3 months for 2 years and then every 6 months from years 3 to 5 . Most routine patient follow-up appointments included a physical examination, laboratory tests (including assessing CA199, CA72-4, and CEA levels), chest radiography, abdominopelvic ultrasonography or CT and an annual endoscopic examination. Overall survival was calculated from the day of surgery until death or until the final follow-up date, whichever occurred first.

\section{Statistical analysis}

All of the statistical analyses were performed using SPSS v. 18.0 for Windows (SPSS Inc., Chicago, IL), and violin plots were generated with the R (v.3.2.5) program. All continuous variables were presented as the mean \pm standard deviation. Chi-square or Fisher's exact tests were used for categorical variables. Cumulative survival rates were compared using the Kaplan-Meier method and log-rank test. Regression analysis was performed using the Cox proportional hazards regression model in multivariate analyses. Values of $P<0.05$ were considered statistically significant.

\section{Results}

\section{Patient characteristics of LG and OG groups}

Table 1 shows the clinicopathologic characteristics of all patients. The characteristics of LG and OG groups were similar except BMI, tumor location and reconstruction method. Compared with OG, LG was associated with more LNs retrieved and reduced intraoperative time, intraoperative blood loss and postoperative morbidity (all $P<0.05$ ). A total of $66.8 \%(571 / 855)$ of the LG patients and $74.0 \%$ $(114 / 154)$ of OG patients had ELNs, but the difference was not significant $(P=0.091)$. The Supplementary Fig. 1 showed that the distribution of LN size was significant different between each pN stage $(P<0.001)$ (Supplementary material 1). The distribution of $\mathrm{pN}$ positivity in SLN or ELN patients was also comparable between the two groups (Supplementary material 2).

\section{The distribution of ELNs in two groups}

The violin plot revealed that the LG and OG groups have the same median diameter of ELNs $(1.3 \mathrm{~cm})$, and the distribution of ELN size was similar between the two groups (Fig. 1). The correlation between the size of LNs detected by CT and the positivity of LNs was showed in the Supplementary Table (Supplementary material 3).The incidence of ELNs in areas I to IV in the LG and OG groups was 23.9 vs. $29.2 \%(P=0.156), 54.9$ vs. $55.4 \%$ $(P=0.710), 46.8$ vs. $49.4 \%(P=0.599)$ and 12.9 vs. $16.7 \%(P=0.630)$, respectively, which was also similar between the two groups.

\section{Surgical quality of LN dissection between the two groups}

Compared with the OG group, the LG group was associated with more retrieved LNs $(31.6 \pm 11.8$ vs. $27.7 \pm 12.3$, $P<0.001)$. For patients with SLN, a stratified analysis revealed that the number of LNs retrieved in LG was also significantly greater compared with patients undergoing OG. Regarding patients with ELN, the number of harvested LNs was similar between the two groups. Further stratified analysis based on the dissected areas revealed that among patients with SLN, patients in the LG group had significantly more LN dissections than patients in the OG group in all four areas. Significantly, more LN dissections were harvested in area II in the LG group, whereas a comparable number of LNs were retrieved in both groups from areas I, III and IV (Table 2). 
Table 1 Patients clinicopathological characteristics between OG and LG

\begin{tabular}{|c|c|c|c|}
\hline \multirow[t]{2}{*}{ Characteristic } & \multicolumn{2}{|l|}{ All patients } & \multirow[t]{2}{*}{$P$} \\
\hline & OG $(n=154)$ & $\mathrm{LG}(n=855)$ & \\
\hline Age, $n(\%)$ & & & 0.457 \\
\hline$<65$ & $88(57.1)$ & $524(61.3)$ & \\
\hline$\geqq 65$ & $66(42.9)$ & $331(38.7)$ & \\
\hline Gender, $n(\%)$ & & & 0.997 \\
\hline Male & $118(76.6)$ & $655(76.6)$ & \\
\hline Female & $36(23.4)$ & $200(23.4)$ & \\
\hline BMI, $n(\%)$ & & & 0.046 \\
\hline$<25 \mathrm{~kg} / \mathrm{m}^{2}$ & $140(90.9)$ & $723(84.6)$ & \\
\hline$\geqq 25 \mathrm{~kg} / \mathrm{m}^{2}$ & $14(9.1)$ & $132(15.4)$ & \\
\hline ASA, $n(\%)$ & & & 0.470 \\
\hline I & $63(40.9)$ & $308(36.0)$ & \\
\hline II & $85(55.2)$ & $517(60.5)$ & \\
\hline III & $6(3.9)$ & $30(3.5)$ & \\
\hline Previous abdominal Surgery(PAS), $n(\%)$ & & & 0.141 \\
\hline None & $141(91.6)$ & $745(87.1)$ & \\
\hline Yes & $13(8.4)$ & $110(12.9)$ & \\
\hline Charlson score, $n(\%)$ & & & 0.558 \\
\hline 0 & $114(74.0)$ & $596(69.7)$ & \\
\hline $1-2$ & $38(24.7)$ & $246(28.8)$ & \\
\hline$\geq 3$ & $2(1.3)$ & $13(1.5)$ & \\
\hline Lymphadenectomy, $n(\%)$ & & & 0.053 \\
\hline $\mathrm{D} 1+$ & $21(13.6)$ & $185(21.6)$ & \\
\hline D2 & $133(86.4)$ & $700(81.9)$ & \\
\hline Enlarged LNs, $n(\%)$ & & & 0.091 \\
\hline Yes & $40(26.0)$ & $284(33.2)$ & \\
\hline No & $114(74.0)$ & $571(66.8)$ & \\
\hline LNs sizes, $n(\%)$ & & & 0.110 \\
\hline$\leq 1 \mathrm{~cm}$ & $40(26.0)$ & $292(34.2)$ & \\
\hline$>1.0, \leq 1.3 \mathrm{~cm}$ & $38(24.7)$ & $179(20.9)$ & \\
\hline$>1.3, \leq 1.6 \mathrm{~cm}$ & $47(30.5)$ & $192(22.5)$ & \\
\hline$>1.6, \leq 1.9 \mathrm{~cm}$ & $11(7.1)$ & $62(7.3)$ & \\
\hline$>1.9, \leq 2.2 \mathrm{~cm}$ & $3(1.9)$ & $46(5.4)$ & \\
\hline$>2.2, \leq 2.5 \mathrm{~cm}$ & $5(3.2)$ & $32(3.7)$ & \\
\hline$>2.5$ & $10(6.5)$ & $52(6.1)$ & \\
\hline Neoadjuvant chemotherapy, $n(\%)$ & & & 0.157 \\
\hline Yes & $9(5.8)$ & $28(3.3)$ & \\
\hline No & $145(94.2)$ & $827(96.7)$ & \\
\hline Adjuvant Chemotherapy, $n(\%)$ & & & 0.065 \\
\hline Yes & $93(60.4))$ & $446(52.2)$ & \\
\hline No & $61(39.6)$ & $409(47.8)$ & \\
\hline Reconstruction, $n(\%)$ & & & $<0.001$ \\
\hline Roux-en-Y & $87(56.5)$ & 425 (49.7) & \\
\hline B-I & $39(25.3)$ & $369(43.2)$ & \\
\hline B-II & $21(13.6)$ & $31(3.6)$ & \\
\hline Esophagogastric & $7(4.5)$ & $30(3.5)$ & \\
\hline Operative time (min) & $261.6 \pm 75.7$ & $171.0 \pm 47.7$ & $<0.001$ \\
\hline Without PAS & $262.4 \pm 75.4$ & $170.0 \pm 47.2$ & $<0.001$ \\
\hline With PAS & $254.2 \pm 81.8$ & $177.5 \pm 50.6$ & $<0.001$ \\
\hline Intraoperative blood loss (ml) & $225.8 \pm 317.5$ & $76.0 \pm 143.5$ & $<0.001$ \\
\hline
\end{tabular}


Table 1 (continued)

\begin{tabular}{|c|c|c|c|}
\hline \multirow[t]{2}{*}{ Characteristic } & \multicolumn{2}{|l|}{ All patients } & \multirow[t]{2}{*}{$P$} \\
\hline & $\mathrm{OG}(n=154)$ & $\mathrm{LG}(n=855)$ & \\
\hline Morbidity, $n(\%)$ & & & 0.016 \\
\hline Yes & $27(17.5)$ & $89(10.4)$ & \\
\hline No & $127(82.5)$ & $766(89.6)$ & \\
\hline Morbidity score, $n(\%)$ & & & 0.230 \\
\hline I-II & $16(10.4)$ & $67(7.8)$ & \\
\hline III-IV & $10(6.5)$ & $21(2.5)$ & \\
\hline $\mathrm{V}$ & $1(0.7)$ & $1(0.1)$ & \\
\hline Gastrectomy extent, $n(\%)$ & & & 0.819 \\
\hline Total & $76(49.4)$ & 425 (49.7) & \\
\hline Distal & $71(46.1)$ & $400(46.8)$ & \\
\hline Proximal & $7(4.5)$ & $30(3.5)$ & \\
\hline Tumor location, $n(\%)$ & & & 0.024 \\
\hline Upper & $50(32.5)$ & $205(24.0)$ & \\
\hline Middle & $16(10.4)$ & 159 (18.6) & \\
\hline Lower & $68(44.2)$ & $398(46.5)$ & \\
\hline$\geq 2$ areas & $20(13.0)$ & $93(10.9)$ & \\
\hline Tumor diameter, $n(\%)$ & & & 0.899 \\
\hline$<2 \mathrm{~cm}$ & $17(11.0)$ & $89(10.4)$ & \\
\hline $2-5 \mathrm{~cm}$ & $64(41.6)$ & $372(43.5)$ & \\
\hline$\geq 5 \mathrm{~cm}$ & $73(47.4)$ & $394(46.1)$ & \\
\hline Pathological margin (mm) & $43.2 \pm 11.0$ & $42.9 \pm 13.1$ & 0.980 \\
\hline Histologic type, $n(\%)$ & & & 0.670 \\
\hline Differentiated & $26(16.9)$ & $147(17.2)$ & \\
\hline Undifferentiated & $128(83.1)$ & $708(82.8)$ & \\
\hline pT stage, $n(\%)$ & & & 0.410 \\
\hline $\mathrm{T} 1$ & $36(23.4)$ & $231(27.0)$ & \\
\hline $\mathrm{T} 2$ & $13(8.4)$ & $93(10.9)$ & \\
\hline $\mathrm{T} 3$ & $45(29.2)$ & $251(29.4)$ & \\
\hline $\mathrm{T} 4 \mathrm{a}$ & $60(39.0)$ & $280(32.7)$ & \\
\hline Harvested LNs & $27.7 \pm 12.3$ & $31.6 \pm 11.8$ & $<0.001$ \\
\hline Metastatic LNs & $5.9 \pm 7.8$ & $5.8 \pm 8.5$ & 0.902 \\
\hline pN stage, $n(\%)$ & & & 0.340 \\
\hline No & $53(34.4)$ & $334(39.1)$ & \\
\hline N1 & $18(11.7)$ & $127(14.9)$ & \\
\hline $\mathrm{N} 2$ & $26(16.9)$ & $120(14.0)$ & \\
\hline N3 & $57(37.0)$ & $274(32.0)$ & \\
\hline pTNM stage, $n(\%)$ & & & 0.564 \\
\hline Ia & $28(18.2)$ & $196(22.9)$ & \\
\hline $\mathrm{Ib}$ & $12(7.8)$ & $76(8.9)$ & \\
\hline IIa & $15(9.7)$ & $91(10.6)$ & \\
\hline IIb & $18(11.9)$ & $90(10.5)$ & \\
\hline IIIa & $14(9.1)$ & $87(10.2)$ & \\
\hline IIIb & $30(19.5)$ & $118(13.8)$ & \\
\hline IIIc & $37(24.0)$ & $197(23.0)$ & \\
\hline
\end{tabular}

ASA American Society of Anesthesiologists, BMI body mass index, TNM tumor node metastasis staging 


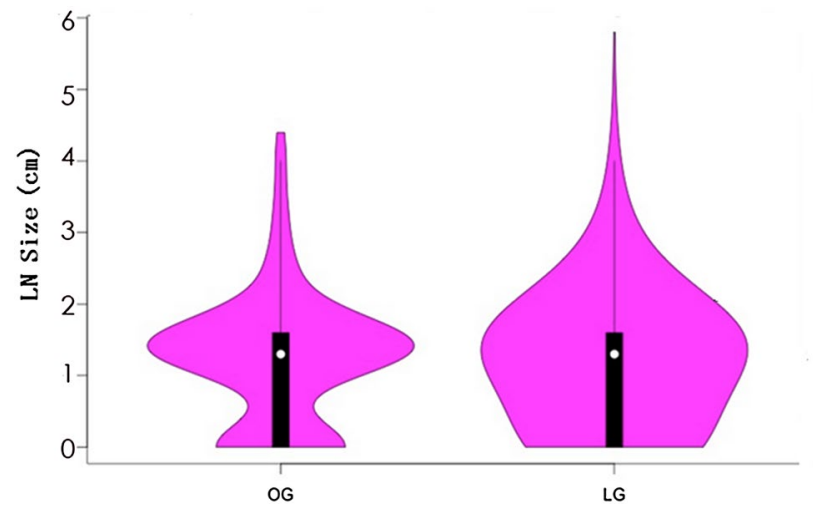

Fig. 1 The violin plot in distribution of ELNs between two groups

\section{Survival analysis between two groups}

Of all patients, 939 (93.1\%) completed a postoperative follow-up, and the median follow-up period was 50 (range 3-76) months. For SLN patients, a stratified analysis revealed no difference in the OS between the two groups $(P=0.867)$; however, the OS was better in the LG group compared with the OG group for ELN patients $(P=0.038)$. Furthermore, we stratified the analysis based on the pN stage. For $\mathrm{pN}+$ patients with ELNs, the OS in the LG group is also superior to that for OG $(P=0.009)$, whereas the $\mathrm{OS}$ is similar between the two groups for $\mathrm{pNO}$ patients with ELNs and pN0 and pN+ patients with SLN (Fig. 2).

Table 2 Lymph nodes dissection between OG and LG

\begin{tabular}{|c|c|c|c|c|c|c|c|c|c|c|}
\hline \multirow[t]{2}{*}{ Characteristic } & \multicolumn{4}{|c|}{ Small LN patients } & \multirow[t]{2}{*}{$P$} & \multicolumn{4}{|c|}{ Large LN patients } & \multirow[t]{2}{*}{$P$} \\
\hline & OG & $n$ & LG & $n$ & & OG & $n$ & LG & $n$ & \\
\hline Harvested LNs & $21.9 \pm 9.1$ & 40 & $29.7 \pm 11.2$ & 284 & $<0.001$ & $29.7 \pm 12.7$ & 114 & $32.6 \pm 12.0$ & 571 & 0.022 \\
\hline \multicolumn{11}{|l|}{ LNs area } \\
\hline I area & $27.2 \pm 12.2$ & 109 & $31.2 \pm 12.1$ & 651 & 0.002 & $30.8 \pm 12.7$ & 45 & $32.8 \pm 10.8$ & 204 & 0.056 \\
\hline II area & $25.3 \pm 10.3$ & 57 & $29.9 \pm 10.9$ & 386 & 0.002 & $29.1 \pm 13.2$ & 97 & $33.0 \pm 12.4$ & 469 & 0.006 \\
\hline III area & $24.9 \pm 11.9$ & 78 & $30.8 \pm 11.6$ & 455 & $<0.001$ & $30.5 \pm 12.1$ & 76 & $32.5 \pm 12.0$ & 400 & 0.195 \\
\hline IV area & $27.6 \pm 12.3$ & 122 & $31.5 \pm 11.9$ & 745 & 0.001 & $28.1 \pm 12.8$ & 32 & $32.2 \pm 12.0$ & 110 & 0.105 \\
\hline
\end{tabular}
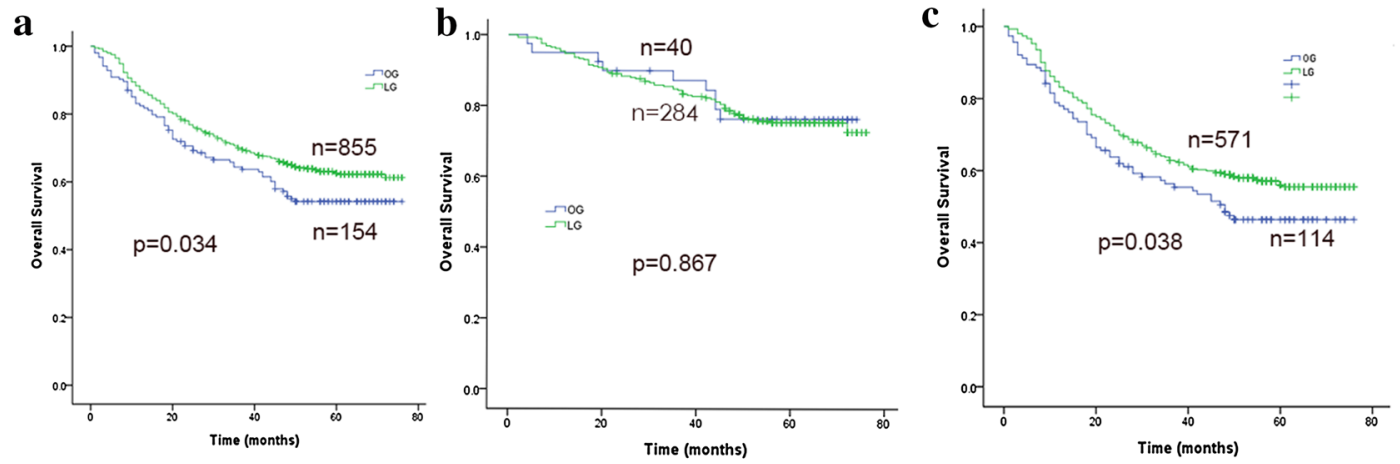
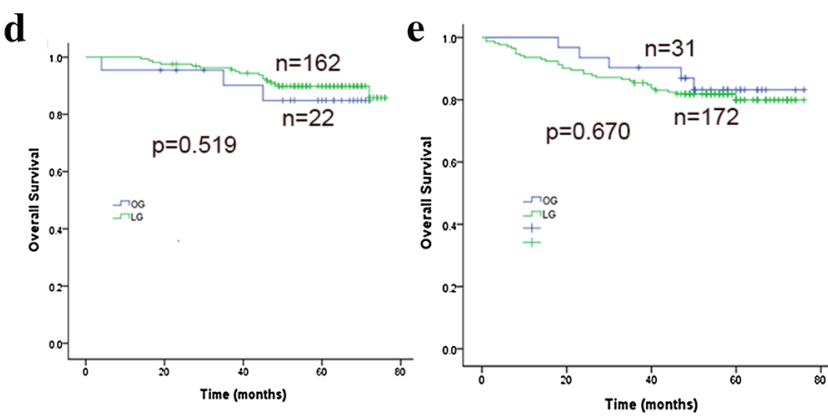

Fig. 2 Overall survival curves for patients underwent LG and OG. a All patient; $\mathbf{b}$ patients without enlarged LNs; $\mathbf{c}$ patients with enlarged LNs; d patents without enlarged LNs in pNO; e patents with enlarged
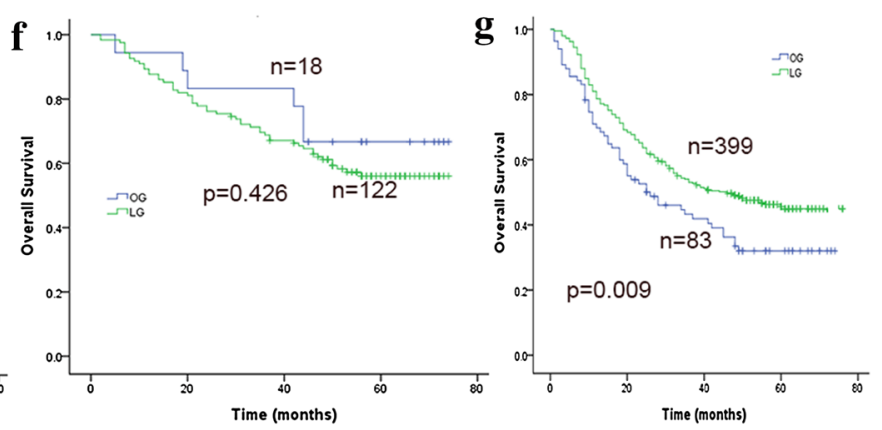

LNs in $\mathrm{pNO}$; $\mathbf{f}$ patents without enlarged $\mathrm{LNs}$ in $\mathrm{pN}+$; $\mathbf{g}$ patents with enlarged LNs in $\mathrm{pN}+$ 
In a forest map analysis of all patients, the actual 3-year OS rate in the LG group was equivalent to the OG group with the exception of those with a Charlson score 1 or 2 and patients without ELNs in area IV. In a stratified analysis of SLN patients, the actual 3-year OS rate was similar between the two groups. In contrast, for ELN patients, the actual 3 -year OS rate in the LG group was significantly increased compared with the OG group with enlarged suprapancreatic LNs (ESLNs). However, the actual 3-year OS rates were similar between the two groups with ELNs in the other areas (Fig. 3). Cox regression indicated that pTNM stage and the degree of LN dissention were independent prognostic factors for patients with ESLNs regardless of undergoing LG (Table 3, Supplementary material 4).

\section{The relation between ESLN size and prognosis between the two groups}

Further stratified analysis based on the different diameters of ESLNs revealed no difference in the actual 3-year OS and cancer-specific survival (CSS)rates between LG and OG in stage I patients with similar LN sizes. For stage II or III patients, the actual 3-year OS and CSS rate in the LG group was significantly increased compared with the OG group for patients with a LN diameter from 1.0 to $1.9 \mathrm{~cm}$ (all $P<0.05$ ). However, if the diameter was greater than
$1.9 \mathrm{~cm}$, the 3-year OS and CSS rate in the LG group gradually declined as the size of ESLN increased, and the rate was significantly reduced compared with OG when the size exceeded $2.5 \mathrm{~cm}$ (Fig. 4, Supplementary material 5). The patterns of initial recurrence showed that the recurrence rate and recurrence model was similar between two groups with similar ESLNs sizes, although LG group seem suffer more locoregional recurrence when the size exceeded $2.5 \mathrm{~cm}$. (Supplementary material 6).

\section{Discussion}

The lymph node stations classification by Japan Gastric Cancer Association is grouped according to the intraoperative relationship between LNs and corresponding vessels. The purpose of our study focuses on the oncologic outcome of LG with OG for gastric cancer with preoperative ELNs. However, for the gastric cancer patients with enlarged or fusional LNs, it cannot completely and accurately confirm them in accordance with the classification of Japan Gastric Cancer Association before operation. Therefore, to better assess the preoperative LNs distribution, we divide the ELNs into I to IV by their anatomical site, which is easy to be evaluated by preoperative CT.
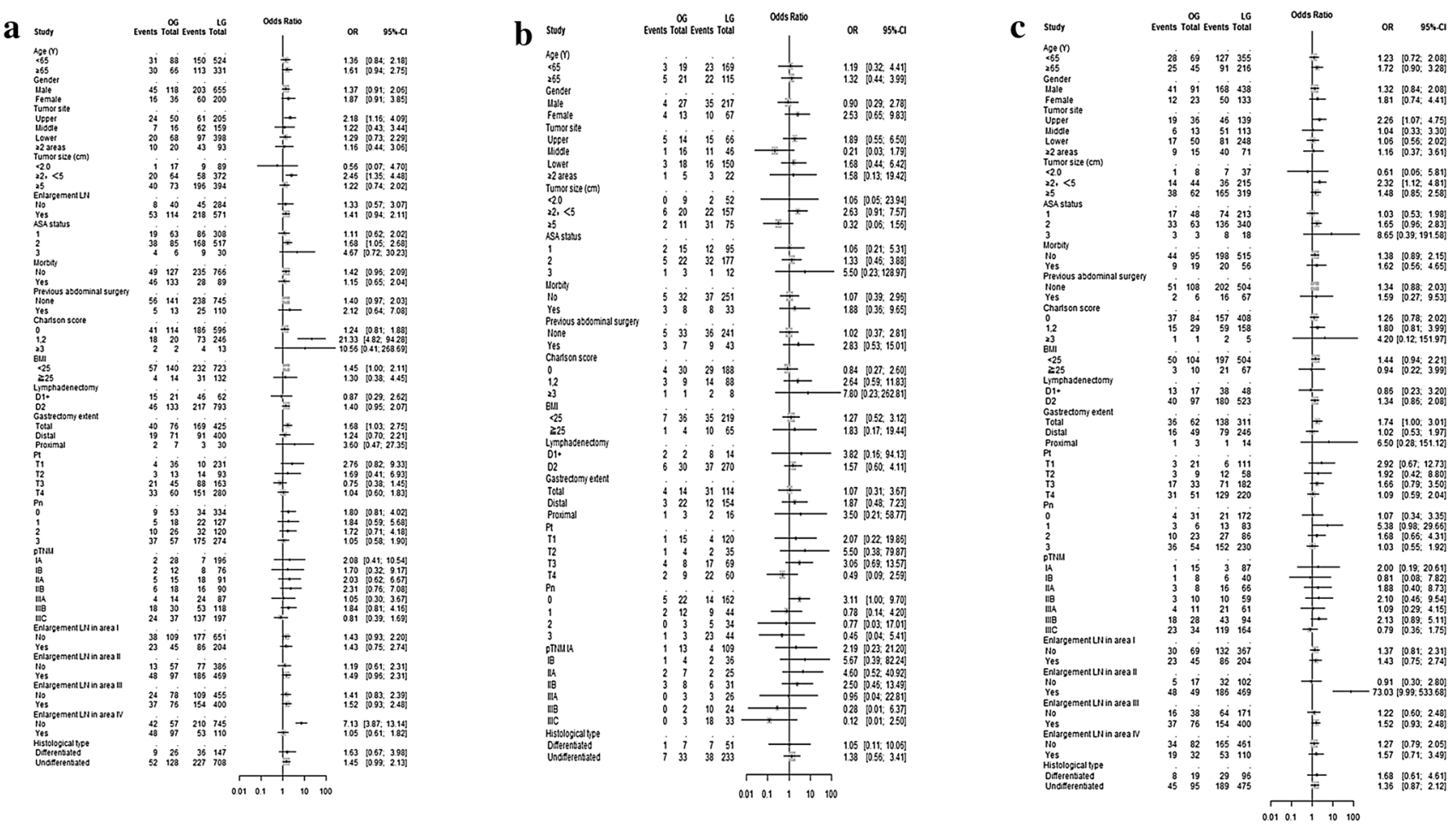

Fig. 3 The forest map in the prognosis of the LG and OG. a All patients, b patients without ELNs, c patients with ELNs. ASA American Society of Anesthesiologists, BMI body mass index, TNM tumor node metastasis staging 
Table 3 Cox regression analysis of survival with ESLNs

\begin{tabular}{|c|c|c|c|c|c|c|}
\hline \multirow[t]{2}{*}{ Characteristic } & \multicolumn{3}{|c|}{ Univariate analysis } & \multicolumn{3}{|c|}{ Multivariate analysis } \\
\hline & HR & $95.0 \% \mathrm{CI}$ & $P$ & HR & $95.0 \% \mathrm{CI}$ & $P$ \\
\hline LG VS OG & 0.716 & $0.520-0.985$ & 0.04 & 0.732 & $0.541-0.990$ & 0.043 \\
\hline Gender & 0.976 & $0.726-1.310$ & 0.869 & - & - & - \\
\hline ASA & 1.415 & $1.058-1.894$ & 0.019 & 1.414 & $1.122-1.782$ & 0.003 \\
\hline Age & 1.073 & $0.802-1.436$ & 0.636 & - & - & - \\
\hline Charlson score & 0.858 & $0.638-1.156$ & 0.315 & - & - & - \\
\hline BMI & 0.841 & $0.567-1.247$ & 0.388 & - & - & - \\
\hline Lymphadenectomy & 1.446 & $0.48-4.351$ & 0.512 & - & - & - \\
\hline Gastrectomy extent & 1.022 & $0.637-1.641$ & 0.928 & - & - & - \\
\hline Reconstruction method & 0.938 & $0.704-1.249$ & 0.66 & - & - & - \\
\hline Tumor location & 1.027 & $0.908-1.163$ & 0.669 & - & - & - \\
\hline Tumor diameter & 1.131 & $0.852-1.501$ & 0.395 & - & - & - \\
\hline pTNM & 1.53 & $1.383-1.691$ & $<0.001$ & 1.563 & $1.440-1.697$ & $<0.001$ \\
\hline Histologic type & 1.085 & $0.767-1.535$ & 0.647 & - & - & - \\
\hline Adjuvant chemotherapy & 0.837 & $0.639-1.095$ & 0.195 & - & - & - \\
\hline
\end{tabular}

ASA American Society of Anesthesiologists, $B M I$ body mass index, TNM tumor node metastasis staging
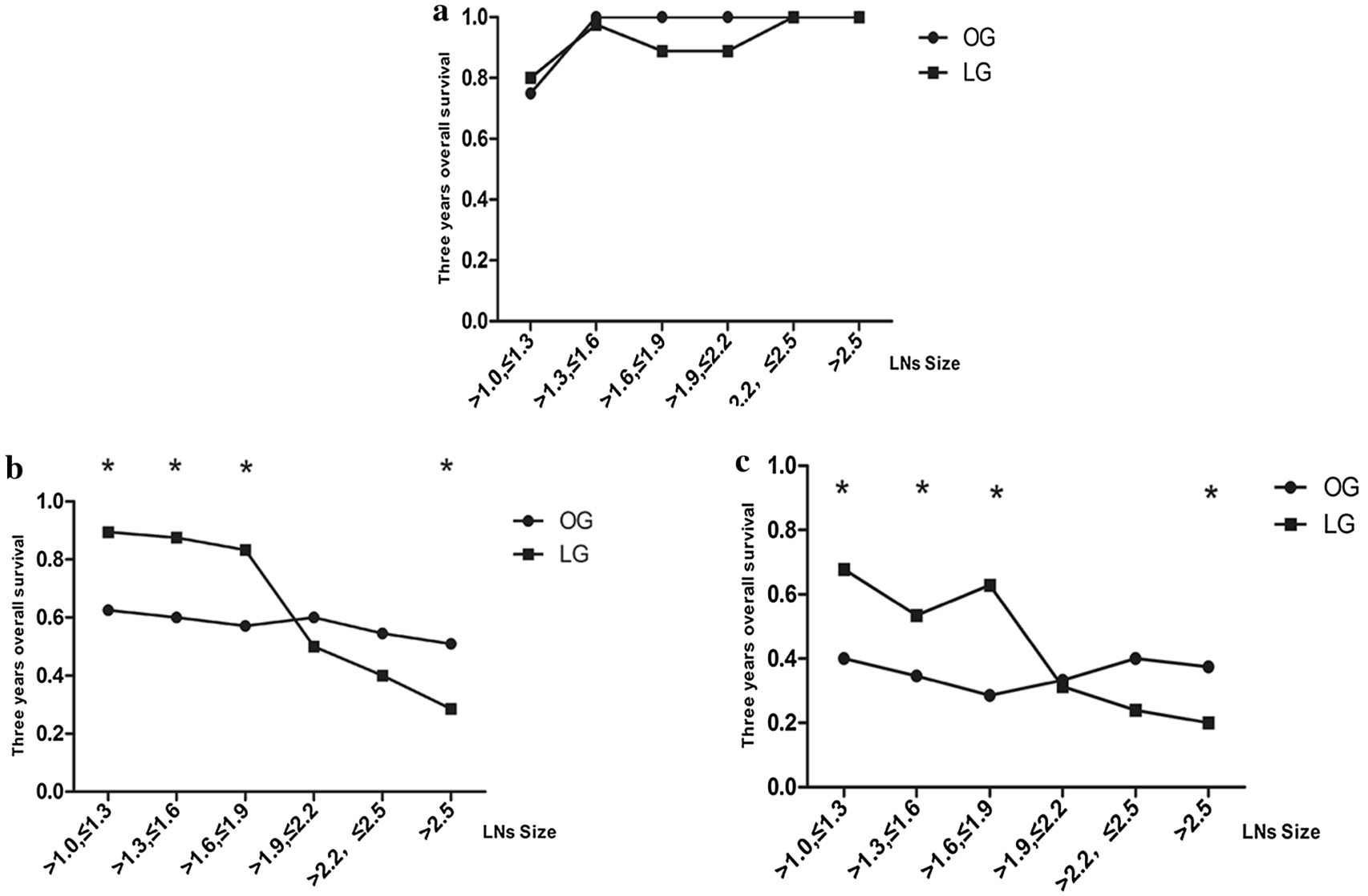

Fig. 43 years overall survival in different diameter of enlarged area II LNs between LG and OG. a pI stage, b pII stage, $\mathbf{c}$ pIII stage

In China, most patients with gastric cancer are diagnosed at an advanced stage, often with enlarged perigastric LNs [22]. The development of techniques, apparatuses and instruments has made it possible for LG to be applied in advanced gastric cancer with satisfactory surgical results. Few studies to date have reported the feasibility of LG for 
advanced gastric cancer. Sato [23] compared 36 cases of laparoscopy-assisted D2 lymph node dissection and 130 cases of open surgery for advanced gastric carcinoma. The results showed that the average number of retrieved lymph node in the laparoscopic group and open surgery group was $(32 \pm 12)$ and $(35 \pm 1)$, respectively, with no statistical significance. Li [24] conducted a randomized controlled trial comparing laparoscopy to open surgery in advanced gastric cancer. The study demonstrated no significant differences between the two groups concerning the number of dissected lymph nodes. There studies suggested that laparoscopy-assisted gastrectomy D2 radical surgery for advanced gastric carcinoma is feasible and safe, and the number of harvested lymph node is the same with open surgery. As with other laparoscopic procedures, there is a learning curve associated with performing LG. Many surgeons are starting to perform this procedure with the tacit acceptance of a lengthy operation time because they often perceive LG to be a complicated technique inevitably subjected to the learning curve effect. A study by Kunisaki et al. [25] focused on one surgeon's surgical learning curve with LG and showed that the operating time was reduced to $230 \mathrm{~min}$ after 60 cases. Our team performed its first LG for early gastric cancer in 2007. After "climbing up" the learning curve, we found that the operation time gradually decreased from our previous study. The average operation time was 237 min after 218 patients [26] and decreased to $196 \mathrm{~min}$ after we completed 1380 cases [27]. Now, the average operation time is only $171 \mathrm{~min}$ in this study. A stable team with a tacit understanding plays an important role in laparoscopic gastrectomy, making the operation easier and faster. With the increasing cases in our center, LG and OG are gradually mature and reach a plateau. While Lymph node dissection and digestive tract reconstruction were relatively fixed and comparable in the two groups, the opening and closing the large abdominal incision in OG involved more time than the assisted small incision in the LG. Therefore, it could be inferred that LG had advantage over OG in terms of operation time. However, it is only the result of our single center, which still needs further evaluation by multicenter data. However, when focusing on comparing the significance of the T classification of LG to OG [11, 12], these studies do not address the differences in efficacy in gastric cancer patients with and without preoperative ELNs. This information is important when deciding the surgical procedures preoperatively based on preoperative examinations. For example, MDCT has more advantages in the judgment of enlarged LNs compared with T classification [28]. Enlarged perigastric lymph nodes complicate the anatomy by pressing or wrapping blood vessels or damaging the local fascia structure. This phenomenon can result in an inaccurate location at the anatomical level or the dissection of blood vessels mistaken for ELNS, thereby increasing the difficulty and risk of LN dissection.
Therefore, the main concern of scholars lies in the curative effect of LN dissections, which is evaluated primarily on the basis of the number of LNs dissected [29]. The results of our study indicate that the total retrieved LNs from LG are significantly increased compared with those from OG and patients with ESLNs. In addition, LG is conducted with significantly less surgery time, reduced blood loss and fewer postoperative complications than OG. Though the rate of $\mathrm{D} 1+\mathrm{LN}$ dissection in the $\mathrm{LG}$ was $21.6 \%$ while that of $\mathrm{OG}$ groups was $13.6 \%$, such difference was not significant. As our study focused on the oncological difference between the two groups with ELN patients, we did not exclude the patients with previous abdominal surgery. Moreover, the further stratification analysis on the influence of the history of abdominal surgery showed that there was no difference in the operation time between the two groups. The amplification effect of the laparoscope could also help surgeons better identify the anatomic space between ELNs and blood vessels, and the surrounding structures by displaying subtler vasculature, nerves and fascia. Moreover, the laparoscopic view provides the surgeon with a better feel of the anatomical complexities when using an ultrasound knife. This feature ensures more refined incisions, more effective hemostasis and less damage to the surrounding tissues, thus enabling the safe dissection of ELNs at the root. For the experienced surgical team, LG achieve better ligation of the blood vessel and complete resection of LNs with ELN, which may explain why more LN were dissected in LG group. The suprapancreatic area is a region with numerous perigastric blood vessels. Furthermore, suprapancreatic LNs are always deep and tend to wrap branches of the celiac artery. Thus, we predict that LG has significant advantages in the dissection of ESLNs. However, in this study, the patients with R1 resection due to pathological margin were excluded. Therefore, the definite safety of the laparoscopic gastrectomy for advanced gastric cancer was unclear.

Long-term survival after surgery is an important aspect of radical gastrectomy. In Japan and South Korea, the proportion of early gastric cancer patients receiving laparoscopic surgery has been growing rapidly. The longterm follow-up conducted by randomized controlled trial (RCT) experiments shows that LG for early gastric cancer can achieve long-term efficacy equivalent to that of OG. Mochiki et al. [30]. reported that they found no significant difference in the five-year survival rate between 89 cases of LG and 60 cases of OG for early gastric cancer, with rates of 98 and 95\%, respectively. The safety and longterm results of LG for advanced gastric cancer have been confirmed by retrospective studies. Multi-center clinical trials regarding long-term oncologic results are also ongoing [24, 31]. However, few comparisons have been made between LG and OG concerning the long-term survival of patients with ELNs. 
Our data show that the 3-year OS rate of LG patients with ESLNs is significantly increased compared with that of OG patients, whereas the OS rate is similar among patients with ELNs in other regions. The stratified analysis of the ESLN diameters shows that among patients with early gastric cancer, no differences in OS and CSS rate were observed between the two groups when the ESLNs are of similar size. For patients with stage II or III cancers, the actual 3-year OS and CSS rate for LG is significantly increased compared with OG when the diameter is between 1.0 and $1.9 \mathrm{~cm}$ (all $P<0.05)$. However, when the diameter further increases, the 3-year OS and CSS rate in LG declines accordingly, and the rate is significantly reduced compared with OG when the size exceeds $2.5 \mathrm{~cm}(P<0.05)$. We suggest that the amplification effect of the laparoscope could help surgeons conduct complete membrane separation in the correct anatomic space and thus ensure the simultaneous resection of both mesentery and lymphatic vessels, which conforms more to the concept of radical operation.

The OS rates of patients with early gastric cancer in the LG and OG groups are similar, even though more lymph nodes are resected in the LG group because the lymph node metastasis rate is low. Although ESLNs are huge, LG could not ensure a better rate of survival even if it could retrieve more LNs, as laparoscopic surgery involves the repeated rolling-over of the gastric wall and ELNs, which might squeeze tumors and ELNs and thus increase the risk of tumor cell dissemination and seeding. Our data showed LG group suffer more locoregional recurrence when the size exceeded $2.5 \mathrm{~cm}$, although it did not reach statistical significance. Based on the discovery above, LG is preferable for patients with advanced gastric cancer when the diameter of ESLNs is between 1.0 and $1.9 \mathrm{~cm}$. In contrast, OG may be a better choice when the diameter of ESLNs is greater than $2.5 \mathrm{~cm}$.

Our data showed that the LN size might become a factor that played a significant role in the decision of whether to perform OG or LG. For advanced gastric cancer patients with ELNs except ESLNs, LG and OG have the same curative effect, both are thus applicable. For patients with an ESLN diameter from 1.0 to $1.9 \mathrm{~cm}$, LG could be chosen preferentially. In contrast, LG is not safe if ESLN are greater than $2.5 \mathrm{~cm}$. For patients with an ESLN diameter from 1.9 to $2.5 \mathrm{~cm}$, both procedures can be chosen. However, the conclusion still needs further confirmation by prospective study. This study has a long follow-up period and a large sample size with actual 3-year OS and CSS rates. Thus, our results are reliable. However, our study still has some limitations. First, being retrospective, this study may have some inevitable hidden bias. Although our data showed that the distribution between LG and OG was similar, the possibility of selection bias when patient decided for open or laparoscopic surgery which could also confound results. Disparities were noted between the number of patients undergoing LG and the number of patients undergoing OG. What's more, due to the lack of relevant data, our study did not compare the socio-economic aspects of the two groups, such as financial burden, which may also have an impact on the results. The study did not include a sufficient number of cases with huge ELNs, i.e., a diameter of greater than $2.5 \mathrm{~cm}$. There may be bias in the results due to $7 \%$ lost to follow-up. These limitations may have influenced the results to a certain extent. Therefore, the exact long-term efficacy is yet to be confirmed but should be studied with a larger sample and a multi-center RCT.

Funding Sponsored by National Key Clinical Specialty Discipline Construction program of China (No. [2012]649), Scientific and technological innovation joint capital Projects of Fujian Province (2016Y9031), General Project of Miaopu scientific research fund of Fujian Medical University (2015MP021), Youth Project of Fujian Provincial Health and Family Planning Commission (2016-1-41), Nature Fund Health Joint Fund Project of Fujian Province (2015J01464).

\section{Compliance with ethical standards}

Conflict of interest None of the authors had any potential conflicts of interest, including relevant financial interests, activities, relationships, and affiliations.

Human rights statement and informed consent All procedures followed were in accordance with the ethical standards of the responsible committee on human experimentation (institutional and national) and with the Helsinki Declaration of 1964 and later versions. Informed consent or a substitute for it was obtained from all patients for being included in the study.

\section{References}

1. Adachi Y, Kamakura T, Mori M, Baba H, Maehara Y, Sugimachi $K$. Prognostic significance of the number of positive lymph nodes in gastric carcinoma. Br J Surg. 1994;81(3):414-6.

2. Fukuda N, Sugiyama Y, Midorikawa A, Mushiake H. Prognostic significance of the metastatic lymph node ratio in gastric cancer patients. World J Surg. 2009;33(11):2378-82.

3. Kutlu OC, Watchell M, Dissanaike S. Metastatic lymph node ratio successfully predicts prognosis in Western gastric cancer patients. Surg Oncol. 2015;24(2):84-8.

4. Kitano S, Iso Y, Moriyama M, Sugimachi K. Laparoscopy-assisted Billroth I gastrectomy. Surg Laparosc Endosc Percutaneous Tech. 1994;4(2):146-8.

5. Katai H, Sasako M, Fukuda H, Nakamura K, Hiki N, Saka M, Yamaue H, Yoshikawa T, Kojima K. Safety and feasibility of laparoscopy-assisted distal gastrectomy with suprapancreatic nodal dissection for clinical stage I gastric cancer: a multicenter phase II trial (JCOG 0703). Gastric Cancer. 2010;13(4):238-44.

6. Kim HH, Han SU, Kim MC, Hyung WJ, Kim W, Lee HJ, Ryu SW, Cho GS, Song KY, Ryu SY. Long-term results of laparoscopic gastrectomy for gastric cancer: a large-scale case-control and case-matched Korean multicenter study. J Clin Oncol. 2014;32(7):627-33. 
7. Kim HH, Hyung WJ, Cho GS, Kim MC, Han SU, Kim W, Ryu SW, Lee HJ, Song KY. Morbidity and mortality of laparoscopic gastrectomy versus open gastrectomy for gastric cancer: an interim report-a phase III multicenter, prospective, randomized Trial (KLASS Trial). Ann Surg. 2010;251(3):417-20.

8. Lee JH, Nam BH, Ryu KW, Ryu SY, Park YK, Kim S, Kim YW. Comparison of outcomes after laparoscopy-assisted and open total gastrectomy for early gastric cancer. Br J Surg. 2015;102(12):1500-5.

9. Seung-Young O, Sebastianus K, Kyung-Goo L, Yun-Suhk S, HwiNyeong C, Seong-Ho K, Hyuk-Joon L, Woo Ho K, Han-Kwang Y. Outcomes of minimally invasive surgery for early gastric cancer are comparable with those for open surgery: analysis of 1013 minimally invasive surgeries at a single institution. Surg Endosc. 2013;28(3):789-95.

10. Toshiyuki K, Naoki H, Souya N, Hisashi N, Michitaka H, Shinya T, Takeshi S, Toshiharu Y. Feasibility and nutritional impact of laparoscopy-assisted subtotal gastrectomy for early gastric cancer in the upper stomach. Ann Surg Oncol. 2014;21(6):2028-35.

11. Ju-Hee L, Min LC, Sang-Yong S, Hoon AS, Do Joong P, HyungHo K. Laparoscopic versus open gastrectomy for gastric cancer: long-term oncologic results. Surgery. 2013;155(1):154-64.

12. $\mathrm{Hu} Y$, Ying $M$, Huang $\mathrm{C}$, Wei $\mathrm{H}$, Jiang Z, Peng $\mathrm{X}, \mathrm{Hu} J$, Du $\mathrm{X}$, Wang B, Lin F. Oncologic outcomes of laparoscopy-assisted gastrectomy for advanced gastric cancer: a large-scale multicenter retrospective cohort study from China. Surg Endosc. 2014;28(7):2048-56.

13. Kelly KJ, Selby L, Chou JF, Dukleska K, Capanu M, Coit DG, Brennan MF, Strong VE. Laparoscopic versus open gastrectomy for gastric adenocarcinoma in the west: a case-control study. Ann Surg Oncol. 2015;22(11):1-7.

14. Adachi T, Eguchi S, Beppu T, Ueno S, Shiraishi M, Okuda K, Yamashita YI, Kondo K, Nanashima A, Ohta M. Prognostic impact of preoperative lymph node enlargement in intrahepatic cholangiocarcinoma: a multi-institutional study by the Kyushu Study Group of Liver Surgery. Ann Surg Oncol. 2015;22(7):1-10.

15. Kendal WS. Lymph node-based prognostics: limitations with individualized cancer treatment. Am J Clin Oncol. 2006;29(3):298-304.

16. Park SR, Kim MJ, Ryu KW, Lee JH, Lee JS, Nam BH, Choi IJ, Kim YW. Prognostic value of preoperative clinical staging assessed by computed tomography in resectable gastric cancer patients: a viewpoint in the era of preoperative treatment. Ann Surg. 2010;251(251):428-35.

17. Edge SB, Compton CC. The American Joint Committee on Cancer: the 7th edition of the AJCC cancer staging manual and the future of TNM. Ann Surg Oncol. 2010;17(6):1471-4.

18. Huang CM, Chen QY, Lin JX, Zheng CH, Ping L, Xie JW, Wang JB, Lu J, Yang XT. Laparoscopic suprapancreatic lymph node dissection for advanced gastric cancer using a left-sided approach. Ann Surg Oncol. 2015;22(7):1.

19. Chang-Ming H, Qi-Yue C, Jian-Xian L, Chao-Hui Z, Ping L, Jian-Wei X, Jia-Bin W, Jun L, Xin-Tao Y. Laparoscopic spleen-preserving no. 10 lymph node dissection for advanced proximal gastric cancer using a left approach. Ann Surg Oncol. 2014;21(6):2051.

20. Chen QY, Huang CM, Lin JX, Zheng CH, Ping L, Xie JW, Wang JB, Lu J, Yang XT. Laparoscopic infrapyloric area lymph node dissection with No. 14v enlargement for advanced lower gastric cancer in middle colic vein approach. Ann Surg Oncol. 2016;23(3):951.

21. Huang CM, Chen QY, Lin JX, Zheng CH, Li P, Xie JW. Huang's three-step maneuver for laparoscopic spleen-preserving No. 10 lymph node dissection for advanced proximal gastric cancer. Chinese. J Cancer Res. 2014;26(2):208-10.

22. Chen W, Zheng R, Baade PD, Zhang S, Zeng H, Bray F, Jemal A, Yu XQ, He J. Cancer statistics in China. CA Cancer J Clin. 2016;66(2):115-32.

23. Sato H, Shimada M, Kurita N, Iwata T, Nishioka M, Morimoto S, Yoshikawa K, Miyatani T, Goto M, Kashihara H, Takasu C. Comparison of long-term prognosis of laparoscopy-assisted gastrectomy and conventional open gastrectomy with special reference to D2 lymph node dissection. Surg Endosc. 2012;26(8):2240-6.

24. Hu Y, Huang C, Sun Y, Su X, Cao H, Hu J, Xue Y, Suo J, Tao $\mathrm{K}, \mathrm{He} \mathrm{X}$. Morbidity and mortality of laparoscopic versus open D2 distal gastrectomy for advanced gastric cancer: a randomized controlled trial. J Clin Oncol. 2016;34(12):1350.

25. Kunisaki C, Makino H, Yamamoto N, Sato T, Oshima T, Nagano Y. Learning curve for laparoscopy-assisted distal gastrectomy with regional lymph node dissection for early gastric cancer. Surg Laparosc Endosc Percutaneous Tech. 2008;18(3):236.

26. Wang JB, Huang CM, Zheng CH, Li P, Xie JW, Lin JX. Efficiency of laparoscopic D2 radical gastrectomy in gastric cancer: experiences of 218 patients (in Chinese). Chin J Gastrointest Surg. 2010;48(7):502-5.

27. Huang CM, Lin JX, Zheng CH, Li P, Xie JW, Lin JX, Wang JB. Clinical efficacy analysis of laparoscopy-assisted radical gastrectomy for 1380 patients with gastric cancer (in Chinese). Chin J Gastrointest Surg. 2012;15(12):1265-8.

28. PhD CYM, PhD Z-GZM, Min YM, Zhang Huan, Zi-Lai Pan MD, PhD JCM, PhD MXM, Ming-Min Chen MD, PhD B-YLM, HaoRan Yin MD. Value of multidetector-row computed tomography in the preoperative $\mathrm{T}$ and $\mathrm{N}$ staging of gastric carcinoma: a largescale Chinese study. J Surg Oncol. 2009;100(100):205-14.

29. Smith DD, Schwarz RR, Schwarz RE. Impact of total lymph node count on staging and survival after gastrectomy for gastric cancer: data from a large US-population database. J Clin Oncol. 2005;23(23):7114-24.

30. Mochiki E, Kamiyama Y, Aihara R, Nakabayashi T, Asao T, Kuwano H. Laparoscopic assisted distal gastrectomy for early gastric cancer: five years' experience. Surgery. 2005;137(3):317-22.

31. Hamabe A, Omori T, Tanaka K, Nishida T. Comparison of longterm results between laparoscopy-assisted gastrectomy and open gastrectomy with D2 lymph node dissection for advanced gastric cancer. Surg Endosc. 2012;26(6):1702-9. 